\title{
A Modeling and Analysis Approach for Mobile Information Systems
}

\author{
Volker Gruhn \\ André Köhler \\ University of Leipzig \\ Department of Computer Science \\ Chair of Applied Telematics / e-Business \\ Klostergasse 3, 04109 Leipzig, Germany \\ Email: \{gruhn, koehler\}@ebus.informatik.uni-leipzig.de
}

\begin{abstract}
This paper introduces a graphical modeling notation based on coloured petri nets for the performance and cost evaluation of mobile applications. When developing such an application some restrictions due to the low bandwith of mobile networks need to be considered. The notation can be used to model the workflow of a mobile application with just a small effort. The resulting model can be (automatically) transformed into a coloured petri net for simulating the communication behaviour depending on typical user interactions. The simulation results are an important basis for improving the applications performance and the occuring costs for using mobile networks.
\end{abstract}

\section{INTRODUCTION}

Since the availability of mobile broadband networks and the reduced costs for mobile devices the use of mobile applications has become an interesting opportunity in several fields. Companies with large divisions of mobile employees (e.g. service technicians, sales representatives, healthcare services) can use mobile applications to gain access to corporate applications and databases at the point of service (POS). Therewith better coordination of mobile employees, rapid task assignment, the avoidance of error-prone format conversion, instant access to customer data and many more becomes feasible [1], [2]. However, until now just a few companies started using these opportunities. The reason for this chary manner is the characteristics of todays mobile networks (in the following used synonym for radio networks, cellular networks, and wireless networks). Unfortunately they hold some unfavourable qualities which are of particular relevance when trying to enable mobile access to a corporate application.

Compared to the familiar LAN environment mobile networks offer just a very small bandwidth. The large data throughput of a typical corporate application often causes long response times under these bandwidth restrictions. Additionally, in mobile networks the upstream bandwidth is often significantly lower than the downstream bandwidth. That works for most consumer applications but causes the break down of corporate applications with frequent push operations (e.g. sending documents generated at POS).

For the use of a mobile network the provider charges a fee on the basis of the time spent online or the data volume transmitted. The todays provider rates are very expensive and often prevent users especially in the consumer area from using mobile applications. Flat rates for mobile data transmission are available since a short time, but often solve the cost problem just for large companies as they can negotiate reasonable prices due the offering of high user numbers and long-term contracts. However, this usually restricts the mobile employee to the use of one network provider. For assuring a preferably permanent network access at the POS the mobile worker should be able to access as many mobile network types and providers as possible. However, the mobile worker will likely not have several flat rate accounts with different network providers.

Within wireless networks all the stability assumptions of the LAN environment are vanishing [3]. It can never be anticipated, if a certain mobile network will be available at a specific place at a specific time. Thus, the time slot for mobile network access as well as the available quality of service (e.g. signal strength, bandwith, costs) are unstable. We assume that in the next five years the number of different mobile networks available at a certain location will grow rapidly especially in urban areas. The increasing number of different mobile network types (e.g. GPRS, EDGE, UMTS, WLAN, WiMAX etc.) combined with some providers can easily lead to the availability of a large number of mobile networks in a certain location, distinguishable in their quality of service.

For a succesful use of a mobile application the circumvention of these shortcomings (long response times, high costs, unstable accessibility) is crucial. As an affected company can not influence these issues in terms of improving network bandwidth, costs and availability it must align the mobile application to the above conditions.

The long response time is caused by small bandwidth due to the large amount of transmitted data. Conversely, the amount of data transmitted must be decreased in order to obtain an improved response time. Thus, an application designated for mobile use need to be evaluated under consideration of the data volume transmitted for typical workflows at the POS, e.g. through simulation. On the basis of these results on the one hand side an application tuning for data intensive communication sequences can be performed, on the other hand side workflow-dependent service levels for mobile networks can be preassigned. Both steps should lead to improved application 
performance through reduced response times.

The high costs arise either from the data volume transmitted or from the time spent online. As time-based rates will be of no relevance in the near future due to the unreasonable high fees and the inscrutable accounting basis, we are focusing on volume-based rates. Obviously, the reduction of the transmitted data volume directly leads to decreasing costs. Again, an evaluation of the mobile application regarding the transmitted data volume for typical workflows at the POS is needed, followed by the same implications as stated above, leading to receded costs for the use of the mobile application.

The unstable accessibility can be improved by providing a technical solution to the mobile worker that allows switching between mobile network types and providers as circumstances demand. The switching between mobile networks while adhering an active network connection is called seamless roaming (see e.g. [4], [5]). This issue is already solved from a technical point of view although the switching between different networks still takes a noticeable amount of time. Today, there are just a few service providers offering software for seamless roaming in case of loosing the current network connection. From a business point of view this technique could be used more efficiently by defining service levels as mentioned above at a workflow level or, even better, at the level of distinct activities or dialogs. Therewith the appropriate bandwidth could be obtained at lowest costs on the basis of single activities. Again, in order to define such service levels the transmitted data volume on the basis of activities is needed.

The three above outlined aspects have one basic issue in common: they depend on the amount of data transmitted over the mobile network. To improve a mobile application regarding these aspects, a detailed analysis of the transmitted data volume is needed. Therefore, we propose the following method: First, a model of the mobile applications' clientside is needed describing the mobile workers' workflow (or business process) in its single steps at the level of network communication respectively application dialogs. Second, an analysis of the model is needed regarding response times and costs. Third, the application need to be readjusted in order to meet defined performance and cost target values. In the following such a method is presented in detail.

The paper is organized as follows: Section II gives an overview about related work. Section III shows detailed requirements for the modeling and the analysis of mobile applications under consideration of the above defined situation. Section IV introduces a petri-net-based graphical modeling notation for the defined purpose. The theoretical work is applied in a case study presented in section V. Section VI gives a short conlusion and shows our planings for further research.

\section{RELATED WORK}

The changes for the discipline of software engineering when developing systems for mobile environments are discussed in [3]. The authors state that "mobility represents a total meltdown of all stability assumptions [...] associated with distributed computing". A comprehensive overview of software engineering for mobile systems is given, regarding issues like models, algorithms, applications and middleware to solve in the future. Our paper adresses some of these modeling issues.

The importance of mobile solutions for improving mobile work processes is shown in several case studies, e.g. for banking services [6], in the paper industry [7], in service organizations [8], in mobile police work [9], in the utility industry [10], and in the construction industry [11], [12].

The general characteristic of mobile work processes is well analyzed in [13]. The authors present a framework to characterise mobile work in order to elicit functional and nonfunctional requirements for a mobile process support system. Furthermore, a lot of aspects regarding the qualities of mobile tasks is presented in [14], [15], [16], and [17].

In [18] and [19] a modeling framework to analyze the performance of applications in a mobile computing environment is presented. UML activity diagrams are used for describing the user interaction with the application as well as the network communication. The paper focuses on different scenarios of physical mobility in order to make code mobility based adaptation policies. Using UML diagrams for modeling mobility aspects is also subject of [20], and [21].

A lot of work is done regarding system architectures and other technical aspects of mobile system. An example for this work is [22], where a three-layer software architecture for distributed and mobile collaboration is presented.

[23] presents an approach for the modeling and performance evaluation of mobile multimedia systems using generealized stochastic petri nets. The author focuses on verifying the optimal performance achievable under some QoS constraints in a given setting of design parameters. In [24] an architectural model that identifies the components representing the essential aspects of a mobile agent system is described.

The interaction design for mobile information systems is subject of [25]. The authors developed a platform that supports the rapid prototyping of multi-channel, multi-modal, contextaware applications and describe how it was used to develop a tourist information system.

In [26] and [27] we showed first results for a cost and performance evaluation of mobile applications. There, we stated that it is quite difficult to obtain meaningful values regarding the usage and the data volume for such applications. For this point, a solution is presented in this paper.

\section{REQUIREMENTS FOR MODELING AND ANALYSING MOBILE APPLICATIONS}

\section{A. Mobile Application Types}

When analyzing the communication behaviour of a mobile application its architecture is of particular relevance. According to [28], mainly four different types can be distinguished. First, a complete offline architecture could be used where no communication via a network occurs. As we focus mainly on network issues, we do not consider this type of architecture. Second, an offline architecture is conceivable where the mobile 
user synchronizes the mobile application occasionally with a central server. Third, a hybrid architecture could combine the advantages of an offline and an online architecture: If a network is available the mobile application communicates with a central server online, if the network is unavailable the mobile application works offline and can be synchronized later with the central server. Fourth, with an always online architecture the mobile application would communicate with a central server exclusively. This architecture is typical for webbased systems. In the following we limit our explanations to the always online architecture for the sake of brevity.

\section{B. Defining Evaluation Objectives}

In order to decide whether a certain application is capable for mobile use, concrete requirements must be defined, most suitable deduced from the mobile workers needs. Therefore the workflow (term used in the following as equivalent for business process) need to be modeled and analysed regarding the use of the mobile application. Thus, the later results can be availed to evaluate the application regarding workflows which e.g. are business critical, have many recurrences, or are important for customer satisfaction. The model should contain at least all steps in the workflow where the mobile application is used. Since the application uses a mobile network, its communication behaviour is of particular interest for the later analysis. Therefore each step in the workflow model should contain detailed information about the communication behaviour of the application which is mainly the data volume transmitted. For a better understanding of these contiguous aspects a short formal description is given in the following.

Be $P$ a set of network providers and $N$ a set of network types. Each combination $(p, n) \in P \times N$ is called wireless network. The volume-based fee $f(p, n)$ for a certain unit for the usage of a network depends on the wireless network $(p, n) \in P \times N$. The maximum upstream bandwidth $b_{u p}(p, n)$ and the maximum downstream bandwidth $b_{\text {down }}(p, n)$ depend also on the mobile network $(p, n) \in P \times N$. A workflow consists of a set of activities $A$. One activitiy $a \in A$ is characterized by the average sent data volume $d v_{\text {sen }}(a)$ an the average received data volume $d v_{r e c}(a)$.

Based on these correlations, certain evaluation objectives can be deduced. For each activitiy $a \in A$ the average request time

$$
t_{a}^{s}(p, n)=\frac{d v_{s e n}(a)}{b_{u p}(p, n)}
$$

as well as the average response time

$$
t_{a}^{r}(p, n)=\frac{d v_{r e c}(a)}{b_{\text {down }}(p, n)}
$$

can be calculated. The overall transmission time of the activitys' data volume is given through:

$$
t_{a}(p, n)=t_{a}^{s}(p, n)+t_{a}^{r}(p, n)
$$

The volume-based fee $c_{a}$ for an activity $a \in A$ can be calculated for the sent data volume with

$$
c_{a}^{s}(p, n)=d v_{\text {sen }}(a) \cdot f(p, n)
$$

as well as the fee for the received data volume:

$$
c_{a}^{r}(p, n)=d v_{r e c}(a) \cdot f(p, n)
$$

The volume-based fee for the overall transmitted data volume for an activity $a \in A$ is given through:

$$
c_{a}(p, n)=c_{a}^{s}(p, n)+c_{a}^{r}(p, n)
$$

For each workflow activity $a \in A$ the average number of recurrences $r_{a}$ can be calculated, e.g. through simulation. Thus, the average costs $c_{w}$ for one workflow recurrence is given by:

$$
c_{w}(p, n)=\sum r_{a} \cdot c_{a}(p, n) \text { for each } a \in A
$$

Therewith seven basic criteria for the evaluation of a mobile application are defined. Of course this model could be extended with more aspects, e.g. allowing the change of the used network between activities, distinguishing costs on the activity level, allowing for volume-dependent fees, and modeling asynchronous communication. As stated above, we focus in this paper on web-based applications and therefore consciously introduced just basic evaluation criteria needed for the understanding of the further explanations. In the following, it is shown how to model the communication behaviour of the application in order to obtain meaningful values for the above defined evaluation criteria.

\section{Petri-net-Based Graphical MODELING NOTATION}

As stated in section III, the modeling of the applications' communication behaviour on the one hand side as well as the mobile workers use of the application (or the workflow) on the other hand side is in scope of our research. Usually, for both aspects concrete data is not available or varies over a certain range. Due to this, we decided to obtain this data through a simulation of both the workflow and the applications' communication behaviour.

For the modelling of workflows plenty of notations are available, but just a few allow the simulation of the created model. Considering this, we decided to use coloured petri nets (CPN), which have a complete formal basis, allow a very flexible modelling, and tools for the modelling and the simulation are available [29], [30]. The advantage of the great flexibility and the excellent expressiveness unfortunately causes also the biggest disadvantages of CPN for our purpose. First, even for small applications the model grows fast and becomes very complex and almost unmanageable. Second, the creation of the model requires extensive knowledge and practice regarding the modeling of a CPN. We do not assume, that the user of our method will have these qualities. Thus, we developed a simple notation that covers the complexity of CPN for our particular purpose. The elements of this notation, needed for modeling web-based applications, are introduced in the following. For the sake of brevity, we give no formal introduction to CPN (see rather [29]), but later CPN examles are explained informal so that also readers having no CPN knowledge will understand their basic functionality. 


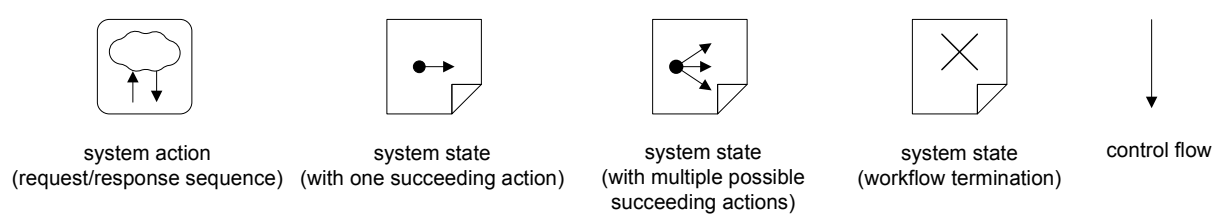

Fig. 1. Notation elements for web-based applications

\section{A. Graphical Notation Elements: Overview}

The notation elements consist of system actions, systems states, and control flow (see Fig. 1). A system action is illustrated by a square with rounded corners describing one request/response sequence via a mobile network, between client and server. A system state is illustrated by a square document symbol as it mostly represents an application dialog. It describes the system state between two system actions where no communication via the mobile network occurs and the mobile worker interacts with the application. The control flow connects system actions and system states, and shows their execution order. A system actions' predecessor(s) as well as the successor(s) must be one or more system states. Equally, a system states' predecessor(s) as well as the successor(s) must be one or more system actions.

\section{B. System Action (request/response sequence)}

For modeling web-based applications just one system action is needed, as communication via the mobile network occurs always as a request/response sequence. The corresponding graphical element contains a cloud representing the network to communicate with (see Fig. 1, utmost left hand side). The starting point of this system action is a system state from which the mobile worker initiates a network request, e.g. through clicking a hyperlink or submitting formular data. In case of clicking a hyperlink, the following response is usually clearly defined and leads to one possible system state (e.g. new webpage). However, in dynamic applications the result may vary over a certain range of defined alternatives depending on the user input. The system action simulates the incidence of one alternative with given probabilities. Furthermore, for both the request and the response it calculates the transmitted data volume from a given range.

This action is defined by a place-bounded CPN as shown in Fig. 2. The used data types and variables in this CPN are defined as follows:

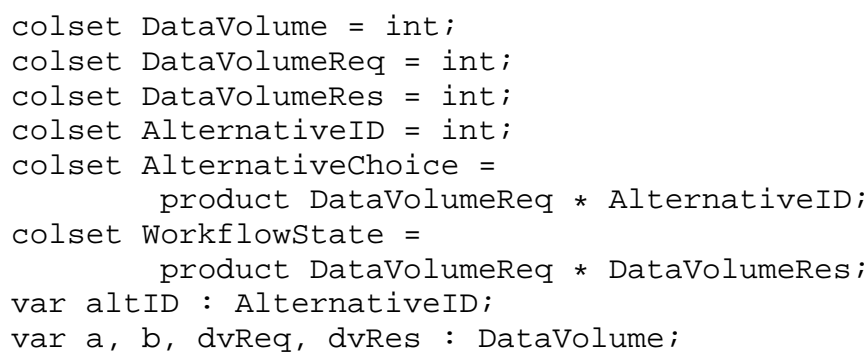

A token in this CPN represents the actual position in the workflow and carries information regarding the transmitted data volume of the current communication sequence. If a token from type WorkflowState is put into place step $A$ the transition network: send and receive fires. In doing so, the function randArcID randomizes from the given alternatives. For each alternative the probability is assigned, the overall sum must be equal to 100. The example in Fig. 2 consists of one alternative with probability 0.8 and a second alternative with probability 0.2 . Furthermore, the function rand $D v$ randomizes the sent data volume $d v_{\text {sen }}(a)$ from a given range. Finally, the transition creates a token as output for place step $B$ carrying the result of the function randArcID as altID and the result of function randDv as $d v$ Req. The place step $B$ has one or more succeeding transitions depending on the defined alternatives. As each alternative has an ID, the token from place $B$ can just be processed by the transition that matches the tokens' altID. The corresponding condition is specified at the outgoing arc of place $B$. The transition alternative $x$ randomizes the received data volume $d v_{r e c}(a)$ from a given range and stores it with the token as $d v R e s$. The token now carries information about the sent data volume $d v R e q$ and the received data volume $d v$ Res and is put into the following place (resulting system state). In Fig. 2 two alternatives are shown, but any number of alternatives is feasible. As we aim on hiding the complexity of CPN, the user can apply this net through the use of the appropriate symbol and need to specify just the variable attributes, which are the actions' name, a range for the requested data volume, the number of alternatives, a probability for each alternative, and a range for the received data volume for each alternative.

\section{System State (with one succeeding action)}

A system state having one possible succeeding system action (e.g. one single hyperlink) does not need to be defined by a CPN. In fact, it matches with one of the source places respectively the drain places from a system action. Such a state is illustrated by a document symbol showing a single arrow (see Fig. 1, second from left hand side).

\section{System State (with multiple possible succeeding actions)}

In most of the cases, a system state will have multiple possible succeeding actions, e.g. a couple of hyperlinks. In this case each succeeding action will be choosen by the mobile worker with a certain probability. Hence, this system state is defined by a CPN as shown in Fig. 3. This system state is illustrated by a document symbol showing some arrows (see Fig. 1, third from left hand side). The used data types and variables in this $\mathrm{CPN}$ are the same as the ones above. The functionality of this CPN is slightly similar as the one explained before. The starting point is a system state, represented by a place (in Fig. 


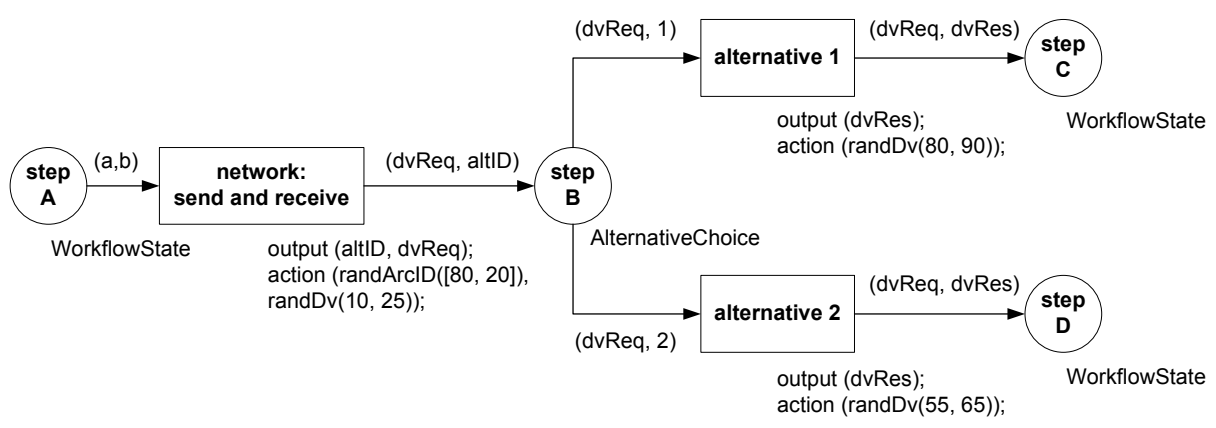

Fig. 2. CPN defining the system action (request/response cycle)



Fig. 3. CPN defining the system state (with multiple succeeding system actions)

3 step A). If a token from type WorkflowState is put into this place the transition local: user choice fires. It randomizes from the given alternatives and puts the resulting altID on the token, placing it into place $B$. From there, only the corresponding transition having the right alternative ID at its incoming arc is able to fire and puts a token in the following place describing the choosen alternative. From there, the corresponding system action could start immediately. In Fig. 3 two alternatives are shown, but any number of alternatives is feasible. As we aim on hiding the complexity of CPN, the user can apply this net through the use of the appropriate symbol and need to specify just the variable attributes, which are the states' name, the number of alternatives, and a probability for each alternative.

\section{E. System State (workflow termination)}

Each workflow has a defined end which is in our case specified through a system state. This state is illustrated by a document symbol showing a cross (see Fig. 1, second from right hand side). This state does not need to be defined by a $\mathrm{CPN}$ as it matches with one of the drain places from the preceding system action.

\section{F. Principles and Guidelines for using the introduced notation}

With the above introduced notation a quite intuitive modeling method is available that allows the creation of CPN describing communication aspects of web-based applications for simulation purposes. Each element of the introduced notation is defined by a place-bounded CPN or by a single CPN place. Thus, with the high-level model an equal CPN is created by connecting the CPN subnets through their bounded places. As web-based applications usually start with the invocation of an URL, the modeled net should start with one system action (URL call) as source element. The workflow should end with one or more defined system states using the workflow termination state. The modeller should avoid describing all possible ways (e.g. links) through an application and rather focus on frequent workflows, abstracting from unusual and less frequent situations.

\section{Case Study}

In the following we show, how the above introduced modeling method was applied for a real application. Therefore, we decided to model an eMail portal. Usually, such portals are designed for the easy access on the users eMail via a notebook or a desktop PC using an internet connection. Furthermore, many people are interested in accessing their mails also when they are away, e.g. using a notebook and GPRS or UMTS adapters. Thus, we would like to answer the question whether such mobile networks have sufficient response times for the given application and what costs occur when using such a mail portal. We decided to use the yahoo mail portal for the further analysis.

The modeling process started as follows. First, we determined typical workflows that are of particular interest for us. That is login and open inbox; compose mail, send and return to inbox; display mail, delete and return to inbox; display mail, reply and return to inbox; and logout. Considering this, we created a model of the yahoo mail portal using the above introduced notation. The result is shown in Fig. 4. The workflow starts with the invocation of the URL (network request/response cycle a.0). The server response contains the data for displaying the login page in which the user enters the login data (system state s.1). By clicking the submit button, the login data is sent to the server (a.1). Two server responses are possible: First, the login failed and the client shows a suitable page (s.3). Second, the login is successful and a mail 


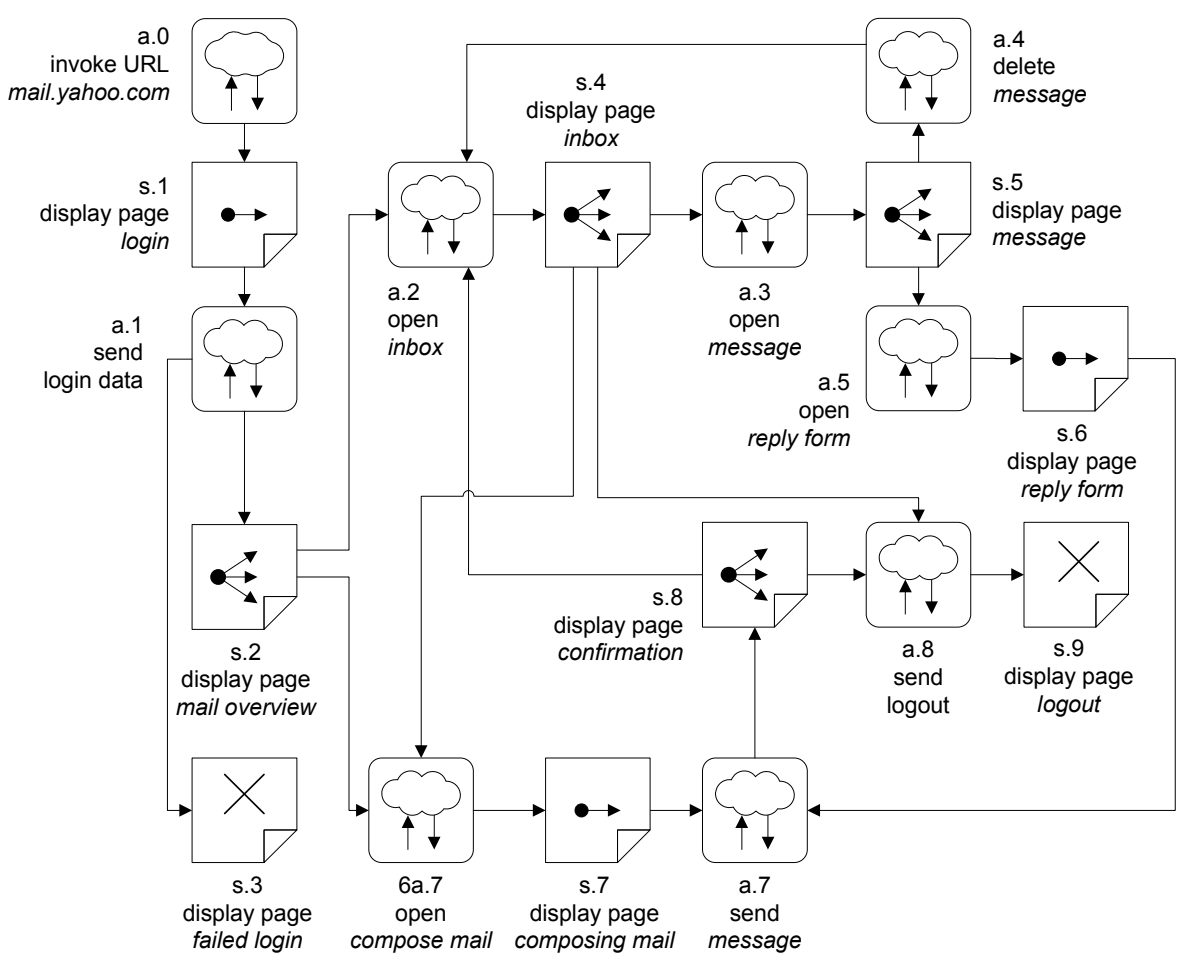

Fig. 4. Yahoo! mail portal

overview page is displayed (s.2). From this point, several user actions are possible. If the user chooses to compose a new mail (a.6), the appropriate page is displayed (s.7). By clicking the send button, the message is sent (a.7) and a confirmation page is displayed (s.8). From there, the user can choose between returning to the inbox folder or to $\log$ out. In the latter case (a.8), a confirmation page is displayed (s.9). From the inbox folder (s.4) a message can be opened (a.3) and gets displayed (s.5). This message can be deleted (a.4), the user then returns to the inbox folder (a.2). The user can also reply to this message (a.5) using a reply form (s.6). The further steps are the same as described in compose mail.

After creating this model, we need to specifiy arc probabilities wherever different alternatives occur and we need to define minimum and maximum values for the expected data volume which is sent and received. We collected the latter data with a HTTP protocol sniffer when using the yahoo mail portal. The resulting data is showed in Table I (values for system actions) and in Table II (values for system states).

The created model can now be transformed into the corresponding petri net according to the above defined subnets. We are currently working on a tool support in order to perform this transformation automatically. The petri net can be used for a simulation of a large number of workflow recurrences. We did such a simulation with CPN-Tools [30] and then analyzed the simulation results regarding the evaluation objectives defined in section III-B. The results are shown in Table III. We conducted the calculation assuming an average up- and downstream bandwidth for three common mobile network types, i.e. GPRS (15 kb/s), UMTS (100 kb/s), and WLAN
$(500 \mathrm{~kb} / \mathrm{s})$. Furthermore, we defined a target response time of two seconds and a maximum response time of five seconds. If a communication sequence' response time lasts up to the threshold value of two seconds, we rated this application piece as mobile usable. If a communication sequence' response time lasts up to the threshold value of five seconds, we rated this application piece as generally mobile usable but with needs for optimization. If a communication sequence' response time lasts longer than five seconds, we rated this application piece as not mobile usable.

The simulation of the net was done 50 times represented by 50 tokens in the CPN. The results show, that considering the above described restrictions the yahoo mail portal is not usable on mobile devices using a GPRS network connection. Using an UMTS network connection, the mail portal works fine in most of the cases. For the actions open message, open reply form, open compose mail, and send message some optimization potential was found. In the WLAN environment, the application performs fine for each system action, as this is the environment the application is originally build for. Considering these results, the application can now be modified, if a use for e.g. UMTS is intended. The results show, that especially the requests for opening a message as well as for opening the reply form would perform badly in most of the cases. The application tuning should start at these points. In the second step we calculated the estimated costs for using the mail portal via a mobile network. We started with the defined workflows which reflect the assumed typical user behaviour. For each workflow we gathered a sequence of system actions from the application model. Based on this 


\begin{tabular}{|c|c|c|c|c|c|c|c|c|c|}
\hline \multirow[t]{2}{*}{ ID } & \multirow[t]{2}{*}{ name } & \multicolumn{2}{|c|}{$\begin{array}{c}\text { request } \\
d v_{\text {sen }} \text { in } \mathrm{kb}\end{array}$} & \multicolumn{3}{|c|}{$\begin{array}{l}\text { response alt. } 1 \\
d v_{r e c} \text { in } \mathrm{kb}\end{array}$} & \multicolumn{3}{|c|}{$\begin{array}{c}\text { response alt. } 2 \\
d v_{r e c} \text { in } \mathrm{kb}\end{array}$} \\
\hline & & $\min$ & $\max$ & prob & $\min$ & $\max$ & prob & $\min$ & $\max$ \\
\hline a.0 & invoke URL yahoo.com & 10 & 20 & 1.0 & 60 & 80 & 0.0 & & \\
\hline a.1 & send login data & 40 & 60 & 0.9 & 80 & 105 & 0.1 & 57 & 95 \\
\hline a. 2 & open inbox & 10 & 20 & 1.0 & 30 & 120 & 0.0 & & \\
\hline a.3 & open message & 10 & 20 & 1.0 & 180 & 260 & 0.0 & & \\
\hline a. 4 & delete message & 10 & 20 & 1.0 & 80 & 160 & 0.0 & & \\
\hline a. 5 & open reply form & 10 & 20 & 1.0 & 180 & 260 & 0.0 & & \\
\hline a. 6 & open compose mail & 10 & 20 & 1.0 & 185 & 185 & 0.0 & & \\
\hline a.7 & send message & 10 & 20 & 1.0 & 90 & 200 & 0.0 & & \\
\hline a. 8 & send logout & 10 & 20 & 1.0 & 30 & 50 & 0.0 & & \\
\hline
\end{tabular}

TABLE I

SPECIFICATION OF SYSTEM ACTIONS

\begin{tabular}{llll}
\hline & & probability of alt. 1 & probability of alt. 2 \\
\hline s.2 & display page mail overview & 0.9 & 0.1 \\
s.5 & display page message & 0.2 & 0.8 \\
s.8 & display page confirmation & 0.8 & 0.2 \\
\hline
\end{tabular}

TABLE II

SPECIFICATION OF SYSTEM STATES

\begin{tabular}{|c|c|c|c|c|c|c|c|c|c|c|c|}
\hline ID & system action & 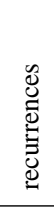 & $\begin{array}{l}\stackrel{\infty}{N} \\
\mathrm{~V} 1 \\
\sim\end{array}$ & 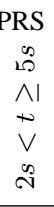 & 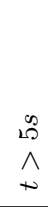 & $\begin{array}{l}\text { N } \\
\text { VI } \\
+\infty\end{array}$ &  & $\begin{array}{l}\infty \\
\stackrel{\infty}{\infty} \\
\wedge\end{array}$ & $\begin{array}{l}\stackrel{\infty}{N} \\
\underset{v}{ } 1\end{array}$ & 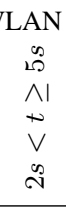 & $\stackrel{\infty}{10}$ \\
\hline a. 0 & invoke URL yahoo.com & 50 & 0 & 3 & 47 & 50 & 0 & 0 & 50 & 0 & 0 \\
\hline a. 1 & send login data & 50 & 0 & 50 & 0 & 50 & 0 & 0 & 50 & 0 & 0 \\
\hline a. 2 & open inbox & 74 & 0 & 0 & 74 & 74 & 0 & 0 & 74 & 0 & 0 \\
\hline a. 3 & open message & 33 & 0 & 0 & 33 & 2 & 31 & 0 & 33 & 0 & 0 \\
\hline a. 4 & delete message & 3 & 0 & 0 & 3 & 3 & 0 & 0 & 3 & 0 & 0 \\
\hline a. 5 & open reply form & 30 & 0 & 0 & 30 & 4 & 26 & 0 & 30 & 0 & 0 \\
\hline a. 6 & open compose mail & 35 & 0 & 0 & 35 & 20 & 15 & 0 & 35 & 0 & 0 \\
\hline a.7 & send message & 30 & 0 & 0 & 30 & 12 & 18 & 0 & 30 & 0 & 0 \\
\hline a. 8 & send logout & 48 & 0 & 48 & 0 & 48 & 0 & 0 & 48 & 0 & 0 \\
\hline
\end{tabular}

TABLE III

Simulation RESUlts

\begin{tabular}{llrrr}
\hline workflow name & $\begin{array}{l}\text { sequence of } \\
\text { system actions }\end{array}$ & $\begin{array}{r}\text { recurrences } \\
\text { per month }\end{array}$ & $\begin{array}{r}\text { average data } \\
\text { volume }(\mathrm{kb})\end{array}$ & $\begin{array}{r}\text { total data } \\
\text { volume }(\mathrm{kb})\end{array}$ \\
\hline login and open inbox & a.0, a.1, a.2 & 60 & 317 & 100489 \\
compose mail, send and return to inbox & a.6, a.7, a.2 & 100 & 450 & 202500 \\
display mail, delete and return to inbox & a.3, a.4, a.2 & 150 & 460 & 211600 \\
display mail, reply and return to inbox & a.3, a.5, a.7, a.2 & 80 & 720 & 518400 \\
logout & a.8 & & 3025 & $\mathbf{1 0 3 6 0 1 4}$ \\
\hline
\end{tabular}

TABLE IV

DEFINITION OF TYPICAL USER BEHAVIOUR 


\begin{tabular}{llrrr}
\hline provider & plan & $\begin{array}{r}\text { base charge } \\
\text { per month (EUR) }\end{array}$ & $\begin{array}{r}\text { charge per } \\
\text { MB (EUR) }\end{array}$ & $\begin{array}{r}\text { inclusive } \\
\text { volume (kb) }\end{array}$ \\
\hline Vodafone & Volume XXL & 69,60 & 0,93 & 512000 \\
Vodafone & Volume XXL 1000 & 104,40 & 0,58 & 1,90 \\
T-Mobile & Data Connect + Data 30 & 15,00 & 1024000 & 30720 \\
T-Mobile & Data Connect + Data 150 & 35,00 & 1,30 & 11,20 \\
\hline
\end{tabular}

TABLE V

MONTHLY COSTS DEPENDING ON DIFFERENT NETWORK PROVIDERS AND PLANS

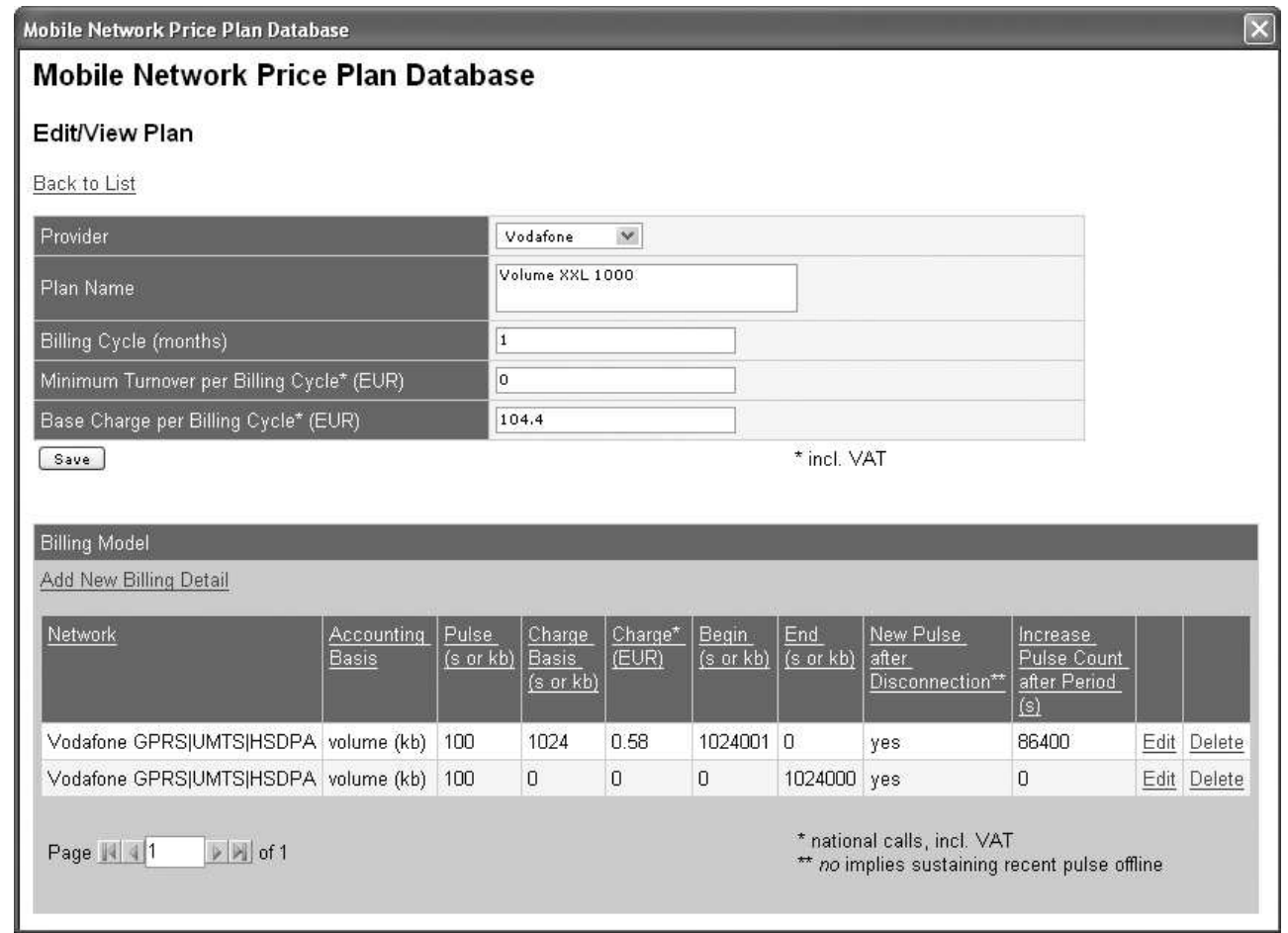

Fig. 5. Mobile network price plan database

information the average request and response data volume for one workflow can be calculated. Furthermore, it must be defined how often the user of the mail portal invokes one of the defined workflows. Table IV shows the results of our user behaviour definition. Assuming this, the user would generate a total data volume of approximately $1 \mathrm{~GB}$ per month.

The last step in the evaluation procedure is the calculation of the resulting mobile network costs. Therefore we developed a database for storing and comparing different price plans. Currently, the databse contains more than 50 plans taken from the German provider market. Fig. 5 shows the detail view for one specific price plan. On the basis of the total data volume per month caused by a user and the price plans the total costs per month depending on the choosen provider and rate can now be calculated. Table $\mathrm{V}$ shows a small extract of such a calculation. For the sake of brevity, we did not not consider time-based plans in the case study as this would led to a more complicated analysis. The example shows, that regarding the given scenario the cheapest plan is the Vodafone Volume XXL 1000 leading to a total of 111,20 EUR per month. Please note that this is not necessariliy the cheapest rate on the provider market as we considered just the four plans for this case study. Furthermore we did not consider the combination of data plans with voice plans or any contract period.

\section{CONCLUSION}

When developing applications for their use with mobile networks, bandwith limitations and occuring costs need to be considered in order to assure the usability of the application at the POS. For already existing applications originally designed for usign in the LAN environment one need to cope with the same challenges when trying to make these applications available at the POS. Therefore, we presented a lightweight graphical modeling notation that can be used for modeling workflows within mobile applications. This notation is based on coloured petri nets and allows the simulation of the created model regarding the frequency of communication sequences as well as their costs and performance. The results of the simulation can be used for aligning the application to business and usability requirements through tuning the application at the right points. 
Further research is planned in several fields regarding the shown method. First, we plan to extend the underlying evaluation model, e.g. through considering different bandwiths for up- an downstreams, allowing asynchronous communication etc. Second, we want to extend also our modeling notation in order to be able to describe more mobile application types, e.g. asynchronous communication with rich clients and offline clients with occasionally synchronization. Third, we work on a tool support for our modeling notation that will allow the automatically transformation of the created model into a CPN. Fourth, we plan to create a catalog of measures helping to optimize mobile applications in general regarding the above stated criteria.

\section{ACKNOWLEDGEMENT}

The Chair of Applied Telematics/e-Business is endowed by Deutsche Telekom AG.

\section{REFERENCES}

[1] V. Gruhn, A. Köhler, and R. Klawes, "Modeling and analysis of mobile service processes by example of the housing industry," in Business Process Management. Springer LNCS 3649, 2005, pp. 1-16.

[2] F. F.-H. Nah, K. Siau, and H. Sheng, "The value of mobile applications: a utility company study," vol. 48 , no. 2. New York, NY, USA: ACM Press, 2005, pp. 85-90.

[3] G.-C. Roman, G. P. Picco, and A. L. Murphy, "Software engineering for mobility: a roadmap," in ICSE '00: Proceedings of the Conference on The Future of Software Engineering. New York, NY, USA: ACM Press, 2000, pp. 241-258.

[4] U. S. Jha, "Wireless landscape need for seamless connectivity," vol. 22, no. 2. Hingham, MA, USA: Kluwer Academic Publishers, 2002, pp. 275-283.

[5] M. Inoue, K. Mahmud, H. Murakami, M. Hasegawa, and H. Morikawa, "Context-based network and application management on seamless networking platform," Wirel. Pers. Commun., vol. 35, no. 1-2, pp. 53-70, 2005.

[6] F. J. M. Borreguero and J. C. Pelaez, "Spanish mobile banking services: An adoption study," in Proceedings of the International Conference on Mobile Business (ICMB'05) - Volume 00. IEEE Computer Society, 2005, pp. 274-280.

[7] V. Kadyte, "Process visibility: How mobile technology can enhance business-customer care in the paper industry," in Proceedings of the International Conference on Mobile Business (ICMB'05). IEEE Computer Society, 2005, pp. 159-165.

[8] D. Innes, S. J. Barnes, and E. Scornavacca, "The impact of wireless field force automation on new zealand trade services organizations," in Proceedings of the International Conference on Mobile Business (ICMB'05). IEEE Computer Society, 2005, pp. 49-55.

[9] D. Pica, C. Sorensen, and D. Allen, "On mobility and context of work: Exploring mobile police work," in Proceedings of the Proceedings of the 37th Annual Hawaii International Conference on System Sciences (HICSS'04). IEEE Computer Society, 2004.

[10] F. F.-H. Nah, K. Siau, and H. Sheng, "The value of mobile applications: a utility company study," Communications of the ACM, vol. 48, no. 2, pp. 85-90, 2005.

[11] A. May, V. Mitchell, S. Bowden, and T. Thorpe, "Opportunities and challenges for location aware computing in the construction industry," in Proceedings of the 7th international conference on Human computer interaction with mobile devices and services. Salzburg, Austria: ACM Press, 2005, pp. 255-258.

[12] S. Bowden, A. Dorr, A. Thorpe, and C. J. Anumba, Mapping site processes for the introduction of mobile IT, ser. eWork and eBusiness in Architecture, Engineering and Construction. London: Taylor \& Francis Group, 2004

[13] C. F. Sorensen, A. I. Wang, H. N. Le, H. Ramampiaro, M. Nygard, and R. Conradi, "The mowahs characterisation framework for mobile work," in Proceedings IASTED International Conference on Applied Informatics, 2002
[14] M. Kakihara and C. Sorensen, "Mobility: An extended perspective," in Proceedings of the 35th Annual Hawaii International Conference on Systems Sciences (HICSS-35'02), 2002, pp. 1756-1766.

[15] P. Luff and C. Heath, "Mobility in collaboration," in Proceedings of the 1998 ACM Conference on Computer supported cooperative work. Seattle, Washington, United States: ACM Press, 1998, pp. 305-314.

[16] D. Pinelle and C. Gutwin, "Designing for loose coupling in mobile groups," in Proceedings of the 2003 international ACM SIGGROUP conference on Supporting group work. Sanibel Island, Florida, USA: ACM Press, 2003, pp. 75-84.

[17] B. Brown and K. O'Hara, "Place as a practical concern of mobile workers," Environment and Planning A, vol. 35, no. 9, pp. 1565-1587, 2003.

[18] V. Grassi, R. Mirandola, and A. Sabetta, "Uml based modeling and performance analysis of mobile systems," in MSWiM '04: Proceedings of the 7th ACM international symposium on Modeling, analysis and simulation of wireless and mobile systems. New York, NY, USA: ACM Press, 2004, pp. 95-104.

[19] V. Grassi and R. Mirandola, "Primamob-uml: a methodology for performance analysis of mobile software architectures," in WOSP '02: Proceedings of the 3rd international workshop on Software and performance. New York, NY, USA: ACM Press, 2002, pp. 262-274.

[20] S. Balsamo and M. Marzolla, "Towards performance evaluation of mobile systems in uml," in The European Simulation and Modelling Conference, 2003.

[21] L. Kloul and J. Kuster-Filipe, "Modelling mobility with uml2.0 and pepa nets," in Proceedings of the Sixth International Conference on Application of Concurrency to System Design. IEEE Computer Society, 2006, pp. 153-164.

[22] S. Dustdar and H. Gall, "Architectural concerns in distributed and mobile collaborative systems," vol. 49 , no. 10-11. New York, NY, USA: Elsevier North-Holland, Inc., 2003, pp. 457-473.

[23] T. Tsang, "Modelling and performance evaluation of mobile multimedia systems using qos-gspn," Wirel. Netw., vol. 9, no. 6, pp. 575-584, 2003.

[24] M. Schoeman and E. Cloete, "Architectural components for the efficient design of mobile agent systems," in SAICSIT '03: Proceedings of the 2003 annual research conference of the South African institute of computer scientists and information technologists on Enablement through technology. , Republic of South Africa: South African Institute for Computer Scientists and Information Technologists, 2003, pp. 48-58.

[25] R. Belotti, C. Decurtins, M. C. Norrie, B. Signer, and L. Vukelja, "Experimental platform for mobile information systems," in MobiCom '05: Proceedings of the 11th annual international conference on Mobile computing and networking. New York, NY, USA: ACM Press, 2005, pp. 258-269.

[26] M. Book, V. Gruhn, M. Hülder, A. Köhler, and A. Kriegel, "Cost and response time simulation for web-based applications on mobile channels," in QSIC '05: Proceedings of the Fifth International Conference on Quality Software (QSIC'05). Washington, DC, USA: IEEE Computer Society, 2005, pp. 83-90.

[27] M. Book, V. Gruhn, M. Hülder, and A. Köhler, "Performance tuning and cost discovery of mobile web-based applications," in Proceedings of the ICWE 2005 Workshop on Web Metrics and Measurement, E. Mendes, Ed. University of Wollongong, School of IT and Computer Science, 2005, pp. 33-37.

[28] M. Book, V. Gruhn, M. Hülder, and C. Schäfer, "A methodology for deriving the architectural implications of different degrees of mobility in information systems," in New Trends in Software Methodologies, Tools and Techniques, M. H. Fujita, Ed. IOS Press, 2005, pp. 281-292.

[29] K. Jensen, Coloured Petri nets (2nd ed.): basic concepts, analysis methods and practical use: volume 1. London, UK: Springer-Verlag, 1996.

[30] http://wiki.daimi.au.dk/cpntools/cpntools.wiki. 